\title{
The LHCb Silicon Tracker, first operational results
}

\author{
D. Esperante*, B. Adeva, A. Gallas, E. Pérez Trigo, P. Rodríguez Pérez, A. Pazos Álvarez, J. Saborido, \\ P. Vázquez \\ Department of Particle Physics, University of Santiago de Compostela
}

\begin{abstract}
A. Bay, M.O. Bettler, F. Blanc, J. Bressieux, G. Conti, F. Dupertuis, V. Fave, R. Frei, N. Gauvin, G. Haefeli, A. Keune, J. Luisier, R. Muresan, T. Nakada, M. Needham, L. Nicolas, M. Knecht, C. Potterat, O. Schneider, M. Tran

École Polytechnique Fédérale de Lausanne
\end{abstract}

\author{
O. Aquines Gutierrez, C. Bauer, M. Britsch, W. Hofmann, F. Maciuc, M. Schmelling, H. Voss \\ Max Planck Institut für Kernphysik, Heidelberg
}

J. Anderson, A. Buechler, A. Bursche, N. Chiapolini, M. de Cian, C. Elsaesser, V. Hangartner, C. Salzmann, S. Steiner, O. Steinkamp, U. Straumann, J. van Tilburg, M. Tobin, A. Vollhardt Physik-Institut der Universität Zürich

\author{
V. Iakovenko, O. Okhrimenko, V. Pugatch \\ National Academy of Sciences, Institute for Nuclear Research, Kiev
}

\begin{abstract}
The Large Hadron Collider beauty (LHCb) experiment at CERN (Conseil Européen pour la Recherche Nucléaire) is designed to perform precision measurements of $b$ quark decays. The LHCb Silicon Tracker consists of two subdetectors, the Tracker Turicensis and the Inner Tracker, which are built from silicon micro-strip technology. First performance results of both detectors using data from Large Hadron Collider synchronization tests are presented.
\end{abstract}

Keywords - Silicon, Tracking, LHCb.

\section{INTRODUCTION}

The LHCb experiment [2] located in the Large Hadron Collider (LHC) at CERN is a single-arm forward spectrometer designed to study rare $b$ decays and CP violation. The LHC is a proton-proton collider working at a bunch crossing rate of 40

MHz. LHCb features excellent tracking and particle identification capabilities and covers an acceptance out to 250 mrad $\times 300 \mathrm{mrad}$ around the LHC beam axis since the $\varnothing$ production of $b$ pairs is peaked a very low polar angle with \& respect to the beam.

The Silicon Tracker (ST) [3] consists of two sub-detectors, the Inner Tracker (IT) and the Tracker Turicensis (TT) which are part of the tracking system [4] of the LHCb experiment. The tracking system consists of the vertex locator system (VELO) placed close to the interaction region, the TT upstream of the LHCb $4 \mathrm{Tm}$ dipole magnet and T1-T3 downstream of the dipole magnet (Fig. 1). VELO and TT use silicon micro-strip detectors. In T1-T3, silicon micro-strips are used in the region close to the beam pipe (Inner Tracker) whereas straw-tubes are employed in the outer region of the stations (Outer Tracker, OT).
The ST gives a total active area of $12 \mathrm{~m}^{2}$ with $\sim 272000$ channels. The same front-end (FE) readout chip (the Beetle [5]) and readout link [6] are used in both the IT and the TT. The data acquisition system is able to read events at a maximum rate of $\sim 1.1 \mathrm{MHz}$. This leads to an output rate of $\sim 337 \mathrm{~GB} \cdot \mathrm{s}^{-1}$ to the Trigger ELectronics and L1 (TELL1) boards [7].

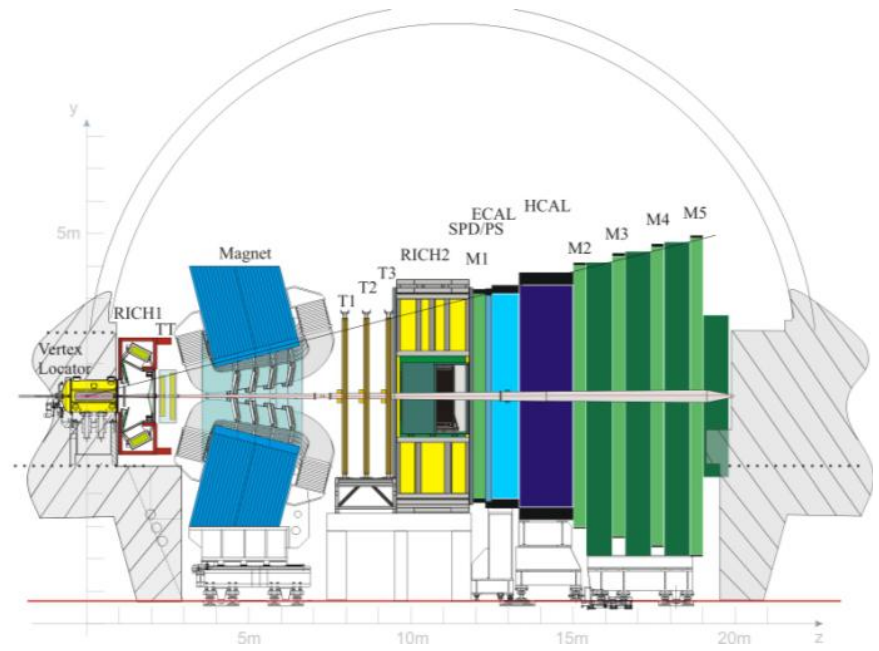

Figure 1. Schematic view of the $\mathrm{LHCb}$ detector

\section{THE DeteCtor DESIGN}

The detector design has been basically constrained by the following considerations:

1) The spatial resolution: simulation studies have shown that a single-hit resolution of $50 \mu \mathrm{m}$ is adequate both for IT 
and TT. Readout strip pitches of about $200 \mu \mathrm{m}$ meet this requirement and were therefore chosen.

2) The hit occupancy: charged particle densities are higher close to the beam pipe. Different readout strip lengths are used for the different regions of the detector to keep maximum strip occupancies at the level of a few percent while minimizing the number of readout channels.

3) Signal shaping time: fast front-end amplifiers with a shaping time of $25 \mathrm{~ns}$ (LHC bunch crossing rate) have to be used in order to avoid pile-up of events from consecutive LHC bunch crossings. Simulation studies have shown that signal remainders $25 \mathrm{~ns}$ after the maximum of the pulse (sampling time of the subsequent bunch crossing) kept below $50 \%$ for the TT and $30 \%$ for the IT are acceptable for the track reconstruction algorithms.

4) The hit efficiency: test beam studies have shown that the hit efficiency decreases rapidly as the $\mathrm{S} / \mathrm{N}$ ratio drops below 10:1. The detector was designed such that a $\mathrm{S} / \mathrm{N}$ ratio in excess of 12:1 can be expected taking into account the expected deterioration from radiation damage.

5) Radiation damage: basic design rules for radiation hard silicon sensors were followed to ensure that the detectors will survive the lifetime of the experiment such that shot noise does not significantly deteriorate the $\mathrm{S} / \mathrm{N}$ performance of the detector and the risk of thermal runaway due to the power dissipated by the leakage currents is avoided.

6) Material budget: the momentum resolution of the LHCb spectrometer is dominated by multiple scattering so the material budget of the detectors had to be kept as small as possible. The TT was designed such that front-end readout electronics and mechanical supports are located outside of the $\mathrm{LHCb}$ acceptance. In the case of the IT, which is located in front of the active region of the OT detectors, a significant design effort was made to keep the amount of material for mechanical supports and for the cooling of front-end electronics as small as possible.

7) Number of channels: the readout electronics is a major contribution to the overall cost of the detector. The largest readout pitches compatible with the required spatial resolution and the longest readout strips compatible with requirements on occupancy and signal-to-noise performance were chosen in order to minimize the number of readout channels.

The Tracker Turicensis (TT) is a $\sim 150 \mathrm{~cm}$ wide and $\sim 130$ $\mathrm{cm}$ height planar tracking station that is placed upstream of the dipole magnet and covers the whole acceptance of the experiment. The TT is housed in a single large box which provides electrical and thermal insulation and is constructed using $500 \mu \mathrm{m}$ thick sensors with a pitch of $183 \mu \mathrm{m}$ and 512 strips. The detector consists of four layers with the strips oriented in $0^{\circ}, 5^{\circ},-5^{\circ}$ and $0^{\circ}$ angles respectively. Fig. 2 shows the layout for four planes. The silicon sensors are bonded together to form readout sectors of up to four sensor with effective strip lengths ranging from $9.4 \mathrm{~cm}$ to $37.2 \mathrm{~cm}$. The granularity of the readout is related to the expected particle occupancy, being higher around the beam pipe, as indicated in the figure with different shadings. The inner readout sectors are connected with up to $56 \mathrm{~cm}$ long Kapton interconnect cables to the respective front-end readout chips located outside the acceptance. In total there are 280 modules and 143000 readout channels.

The Inner Tracker (IT) covers a $\sim 125 \mathrm{~cm}$ wide and $\sim 40 \mathrm{~cm}$ height cross-shaped region (Fig. 3) in the center of the three planar tracking stations downstream of the magnet and close to the beam pipe. Each of the three IT stations comprises four detection layers ( 2 with $\pm 5^{\circ}$ stereo angle) arranged in four boxes which provide electrical and thermal insulation. Although it covers only $1.5 \%$ of the surface area $30 \%$ of the charged tracks pass through it. In the boxes above and below the beam pipe single-sensor modules with a length of $10.8 \mathrm{~cm}$ and $320 \mu \mathrm{m}$ thickness are employed. Two sensor modules with $21.6 \mathrm{~cm}$ and a thickness of $410 \mu \mathrm{m}$ are used in the side boxes. The sensors have a pitch is $198 \mu \mathrm{m}$ and 384 readout strips. In total there are 336 modules and 129000 readout channels.

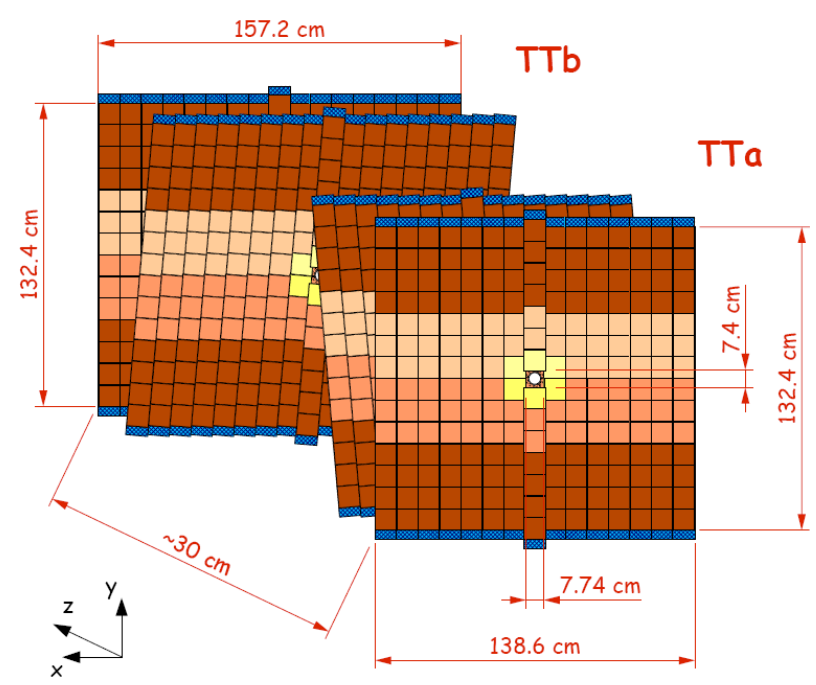

Figure 2. The TT station.

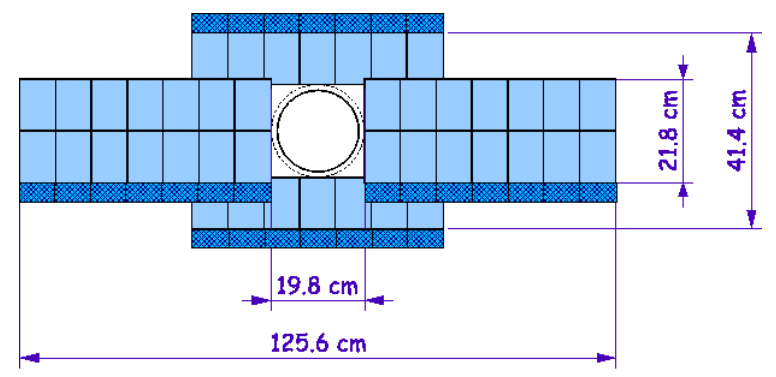

Figure 3. Scheme of an IT x-layer ( $0^{\circ}$ angle $)$

\section{Module Design, Production And Testing}

The module design and production is described in detail in [8] and references therein. The p-on-n silicon strip sensors (Hamamatsu Photonics) are bonded together to give readout strips up to $37.2 \mathrm{~cm}$ in length. Schematics of the detector modules are depicted in Figure 4. The large load capacitance to 
the Beetle preamplifier resulting from long readout strips and interconnect cables requires thicker sensors in order to get larger signals that fulfill the detector $\mathrm{S} / \mathrm{N}$ necessities.

Quality assurance played an important role at all stages of the module production. The silicon sensors were qualified at the factory and re-tested upon reception at the production sites. Tests against infant mortality were performed on the modules at various steps during the assembly; the tests comprised temperature cycling and electrical burn-in for up to $48 \mathrm{~h}$. Shorted and open channels were spotted using the internal Beetle test pulse by analyzing both the strip noise and pulsed signal level. Pedestal runs with the detector box opened allowed pinholes to be identified. The light shining onto the sensors generates leakage currents in the silicon bulk, which, in presence of a pinhole, saturates the front-end pre-amplifier. Metrology measurements were performed before and after assembly of the detector modules.
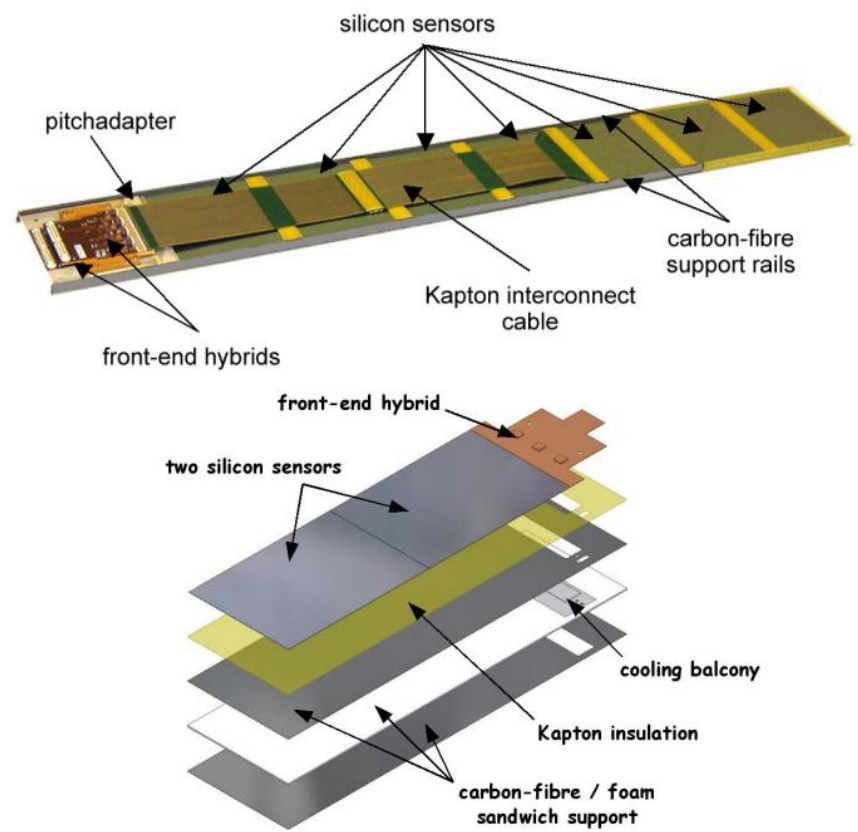

Figure 4. The TT (top) and IT (bottom) detector modules schematics.

\section{THE SILICON TRACKER ElECTRONICS OVERVIEW}

The readout electronics [6] is made up of three building blocks (Fig. 5):

1. The front-end readout electronics, the Beetle chip.

2. The Service Boxes.

3. The TELL1 processing boards.

The silicon sensors are readout by the Beetle chip. This is a radiation hard device placed on a readout hybrid, bonded to the silicon sensors. The chip performs a fast amplification and shaping of the input signals and stores them in an analogue pipeline which will be readout with a fixed latency of $4 \mu \mathrm{s}$. The analogue output data consists of 128 channels plus 16 headers which are digitized in the Service Box. The Beetle output data is transmitted in serial mode over four different ports to service boxes located close to the detector. These house the FE digitizing and control electronics is located close to the detector and hence rated for radiation tolerance ( $\sim$ krads). The Digitizer Boards perform the 8-bit digitization, the serialization using the CERN Giga-bit Optical Link (GOL) ASIC and optical transmission of the Beetle data to the TELL1 boards. In addition, a Control Board [9] is housed in each Service Box. This board carries out the slow and fast control of the FE electronics: it plays the role of configuring the FE devices, performs the monitoring of the temperature and humidity signals and drives the Timing and Trigger Control signals coming from the Timing and Fast Control (TFC) system [10] to the hybrids and Digitizer Boards.

The last stage is the TELL1 real-time processing board. The data from the Digitizer Boards are deserialized and further processed in the TELL1 boards which are placed behind a shielding wall, $60 \mathrm{~m}$ from the interaction point to minimize the amount of electronics exposed to radiation.

The whole data acquisition path operates synchronously with the LHC machine $40 \mathrm{MHz}$ bunch crossing rate. To preserve synchronization over large distances $\mathrm{LHCb}$ has a global clock and fast signal transmission network, the TFC system. The TFC is driven by the Readout Supervisor [11], responsible for distributing the LHC clock and scheduling the trigger decisions.
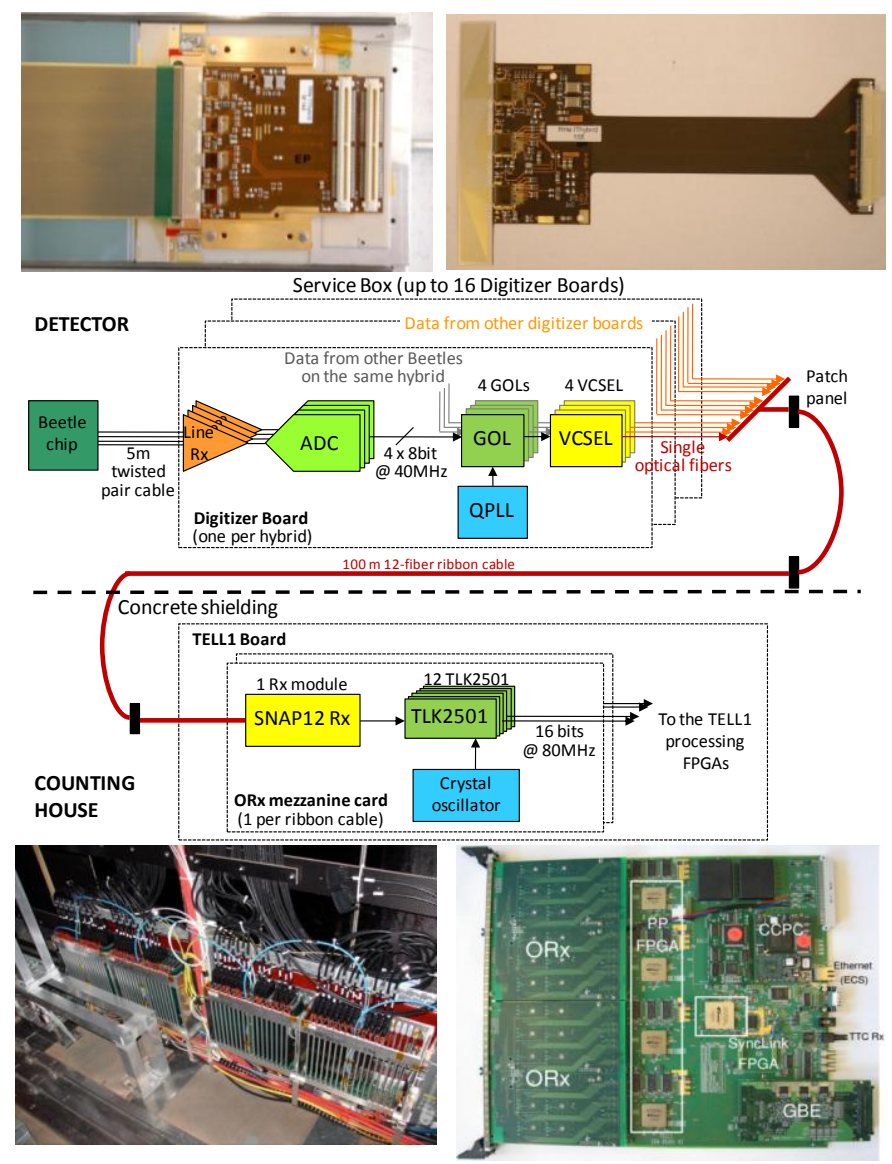

Figure 5. The top pictures from left to right are the TT and IT hybrids housing the Beetles. The middle picture is the scheme of the readout link. The bottom left picture shows the four service boxes corresponding to an IT halfstation and the right one is the ST TELL1 board. 


\section{COMMISSIONING AND STATUS}

The installation of the ST in the LHCb cavern was complete by early summer 2008 (Fig. 6). At the time of writing $99.7 \%$ of the channels in both the IT and TT are functional. Commissioning with particles started after the installation. The ST took part in the global LHCb cosmic running and the LHC synchronization described in the next section. The LHC synchronization tests during 2008 and 2009 provided valuable amounts of data that have been used to time and spatial alignment of the detector as described next. In addition, this running allowed several faults to be identified and repaired.
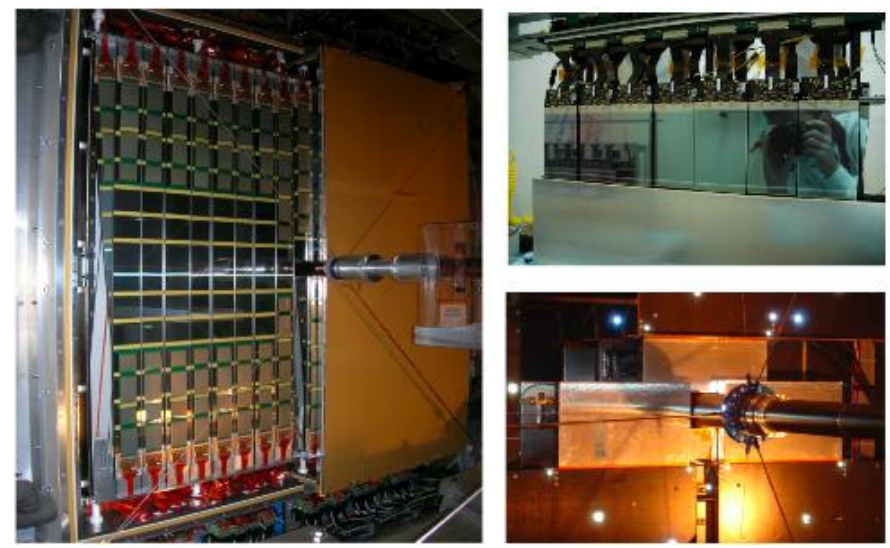

Figure 6. View of the TT installed in LHCb (left). View of the first IT station (bottom right), and detail of one of the detector boxes (top right)

\section{OPERATION With PARTICles: PERFORMANCE STUdieS}

In August 2008, June and October 2009, during the start-up of the LHC, the machine carried out several synchronization tests. In these tests a proton beam of $450 \mathrm{GeV}$ extracted from the SPS (Super Proton Synchrotron) was dumped onto a beam stopper (the 'TED') in the SPS-LHC transfer line, $350 \mathrm{~m}$ behind $\mathrm{LHCb}$. Monte Carlo simulations indicate that most of the particles produced in the TED, which reach the ST, are 10 $\mathrm{GeV} / \mathrm{c}$ muons. During these tests the particle multiplicity was measured to be about 20 times higher than that expected during normal running. The high multiplicity makes the reconstruction of individual tracks in the Silicon Tracker challenging.

\section{A. Time Aligment}

The sampling of the sensor signals must be done with the right timing, i.e. maximizing the signal amplitude. The timing of the $40 \mathrm{MHz}$ sampling clock can be adjusted per service box with a resolution of 104 ps. The electronics has been designed such the maximum clock skew between service box and Beetles is $\pm 1 \mathrm{~ns}$ as this is the tolerance of the clock drivers. In addition the delay of the FE trigger signal can be tuned in $25 \mathrm{~ns}$ steps (the $40 \mathrm{MHz}$ clock period) up to 15 cycles.

In order to perform the internal time alignment of the detector runs were taken for both the IT and TT. First of all the detector had to be coarse time aligned to match the trigger signal with the detector sampling to the right bunch crossing. This was done by reading out 15 consecutive clock cycles and searching for the one with the highest occupancy. After this a fine time alignment of the sampling clock was performed. The procedure consisted in getting data with different delays focusing around the maximum of the signal. The acquired data was averaged for each set of ladders grouped in one front-end service box and a function with the form of a $2^{\text {nd }}$ order CR-RC shaper was used for the fit. The most probable value of the signal determined the optimum sampling point. The delay values for the subsequent data takings were then chosen as the one that maximized the most probable signal amplitude (Fig. 7). After this procedure the detector is internally time aligned with a precision of $\sim 1 \mathrm{~ns}$.
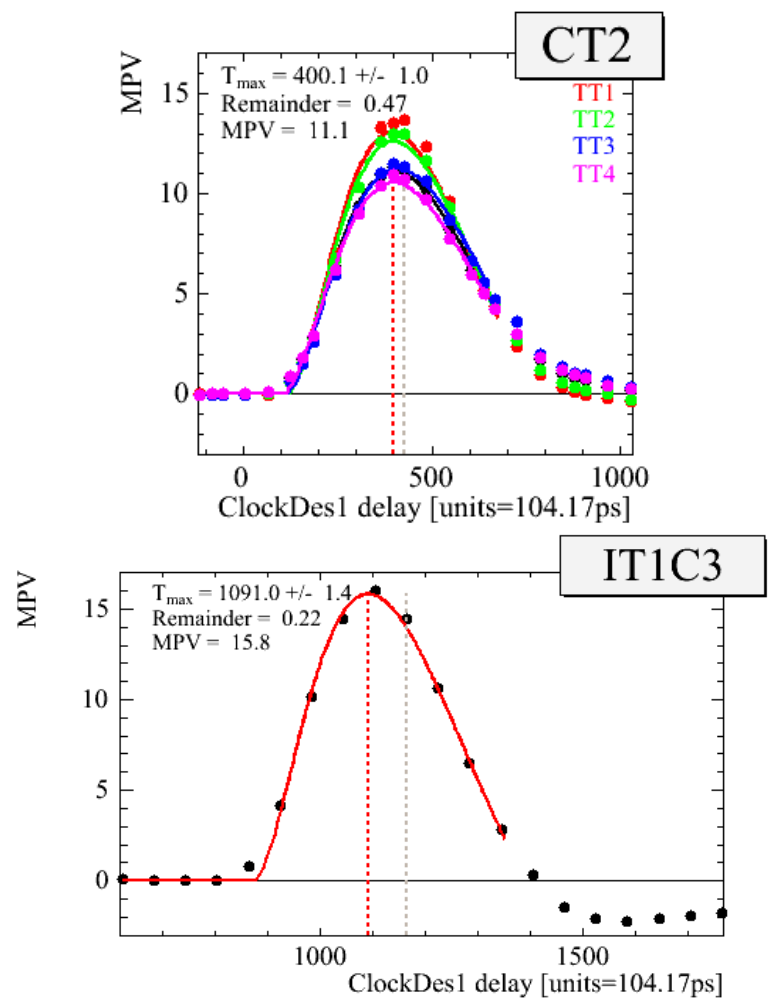

Figure 7. The most probable (MPV) of the sampled charge deposition in the silicon for different timing settings obtained in the TED run. The fit is to the expected form of a CR-RC shaper.IT1C3 and CT2 refer to one IT and one TT service boxes respectively. The labels of different curves in CT2 indicate the number of silicon sensors that conform the module, there the different signal shapes.

\section{B. Spatial Aligment}

A partial survey of the IT detector was performed during commissioning from which a precision in the measurement of $\sim 1-2 \mathrm{~mm}$ for the boxes of $\sim 50 \mu \mathrm{m}$ for the $\mathrm{x}$-layers and ladders was obtained. The positioning of stereo layers could not be measured as they are hidden by the external x-layers. The photogrammetry technique has been used for the box positioning and theodolite measurements for the layers and ladders [12]. The survey measurements were analyzed and the results used in the $\mathrm{LHCb}$ software to provide an initial geometry for the IT detector.

During the TED runs the track density was too high for standard track finding algorithms. Therefore a robust stand-alone track reconstruction algorithm was developed for the IT. The first step in this algorithm is to pick pairs of hits in 
the first and last stations of the IT. A third hit in station 1 or 2 is then required to confirm the track candidate. Next hits are searched for in the middle station. The observed residual distributions (Fig. 8) are used to adjust the position of the Inner Tracker boxes and layers in the x-direction and to verify the correctness of the survey data. Reconstruction with the nominal detector geometry results in an RMS of $310 \mu \mathrm{m}$, by including the information from a partial survey this number is reduced to $270 \mu \mathrm{m}$. After applying corrections at the box and layer level the position of the $\mathrm{x}$-measuring ladders is known to a precision of $100 \mu \mathrm{m}$ or better [13]. The width of the residual distribution is given by the combination of the intrinsic detector resolution, misalignments and the effect of multiple scattering.

After the adjustment of the boxes and layers described in the previous step the pattern recognition provides tracks that are of reasonable quality and allow a full detector alignment to be performed using the standard LHCb alignment tools [14]. For this study the lowest occupancy TED events were selected and used to improve the IT internal alignment using the standalone track reconstruction algorithm and the $\mathrm{LHCb}$ standard track-fitting software [15]. Using this sample the detector boxes were aligned for translations in $\mathrm{X}$ (horizontal), $\mathrm{Y}$ (vertical) and rotations about the beam axis $(Z)$. The detector layers were aligned for $\mathrm{X}$ translations and $\mathrm{Z}$ rotations and the detector ladders for $X$ translations. The validation of the procedure and quality of the alignment has been done using an independent data sample from the TED run and looking at the mean (bias) and $\sigma$ (resolution) of the unbiased residual distributions per ladder. Misalignments still remain and a quantitative approximation of their size can be judged from the $\sigma$ of the bias distribution in Fig. $9(\sim 20 \mu \mathrm{m})$.

The performance of the detector has been assessed using clusters on the reconstructed tracks. The signal to noise ratio (S/N) has been studied for each detector ladder. A fit of a Landau convolved with a Gaussian has been made to the pulse height distribution to extract the Most Probable Value. Fig. 10 shows the obtained $\mathrm{S} / \mathrm{N}$ per ladder. For the long (short) ladders a S/N of 14.5 (15.5) was obtained, consistent with the expectations from the test-beam. The efficiency of the ladders has been measured to be $\sim 98 \%$ using tracks. Most of the ladders with low efficiency are located close to the edge of the detector. Therefore, this number should improve when the detector alignment and global positioning is better understood.

In the case of the TT station, a stand-alone tracking algorithm is not possible because there are only four layers in the detector. Performance studies for this detector rely on track seeds either from the Vertex Locator (VELO) detector or the IT. In the case of the VELO, the overlap with the TT for the tracks coming from the TED is very small region around the beam pipe. This is the reason why in the case of the TT the performance studies are less advanced.

VELO and IT tracks have been extrapolated to the TT and residual to hits have been plotted. Clear correlations have been seen and the quality of the geometric survey performed during the detector installation has been confirmed. As an example Fig. 11 shows the residuals between clusters in the TTb x-layer and the IT Bottom box.
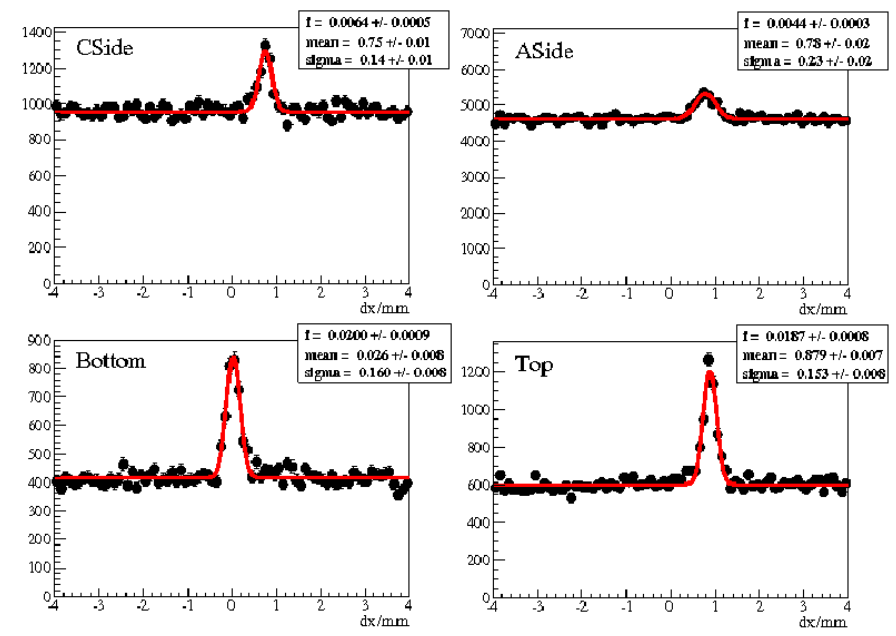

Figure 8. Residual distribution in station 2 for each box with TED events and using the survey data. The peaks correspond to the correct combination of hits, and the smallest peak is for the A-Side where the occupancy is highest. The offsets for the four boxes are obtained by fitting a Gaussian combined with a flat background.

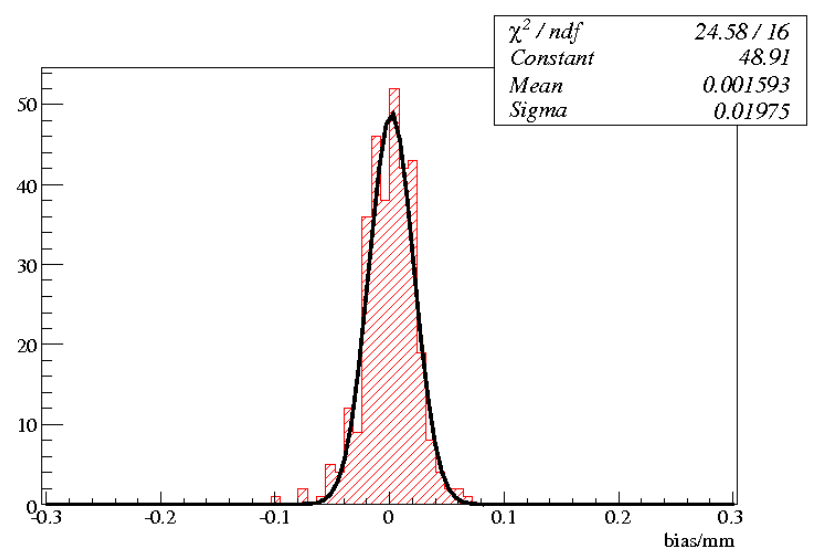

Figure 9. Bias of the unbiased residual distributions per ladder after alignment.

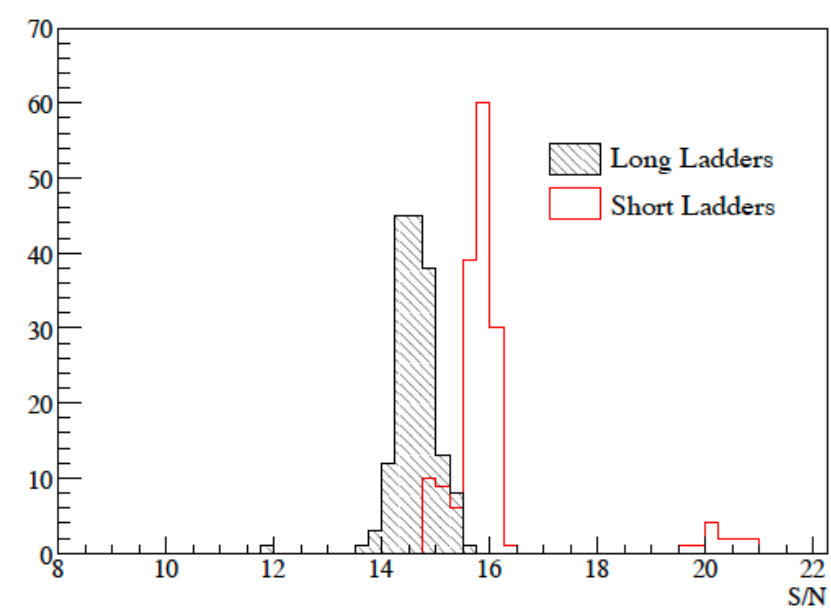

Figure 10. Signal to noise ratio (S/N) of the Inner Tracker ladders. 


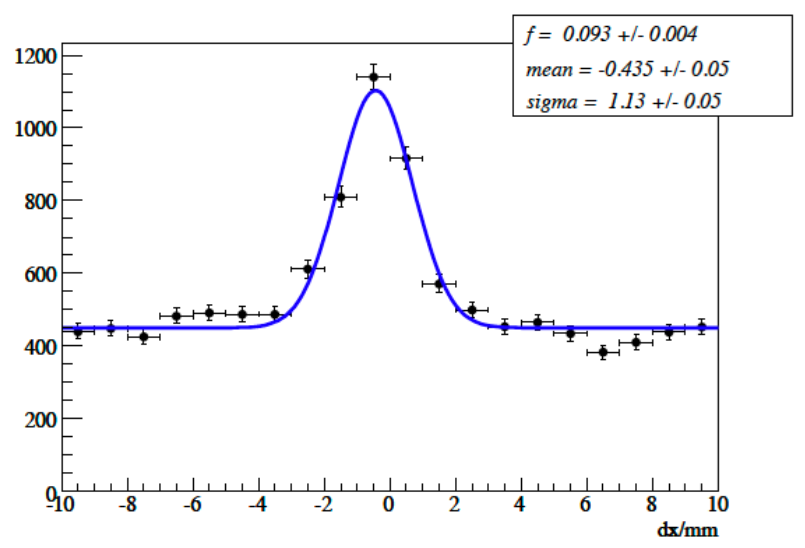

Figure 11. Residual of clusters in the TTb X layer to IT Bottom tracks. The Data was fitted to a Gaussian combined with a flat background.

During November 2009 the LHC beams restarted circulating and first collisions were produced. The LHC and the $\mathrm{LHCb}$ experiment have been running stably during this period and first collision data has been collected. The first analysis of the data has confirmed that the $\mathrm{S} / \mathrm{N}$, the efficiency and the alignment of the detector is consistent with the results from the TED runs. Fig. 12 shows a snapshot of the event display with hits in the tracking stations during the collisions.

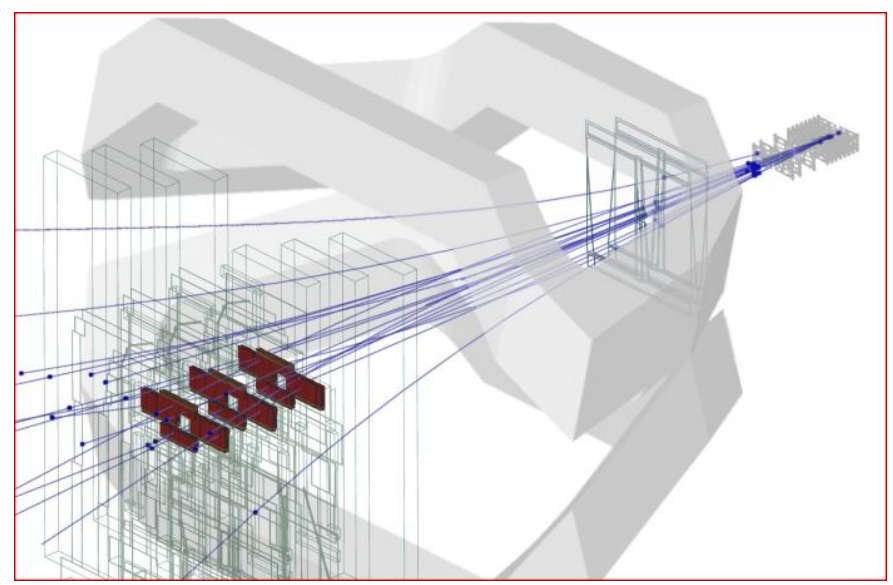

Figure 12. Event display showing hits in the tracking stations.

\section{SUMMARY}

The ST group has designed and developed a silicon micro-strip detector that has been installed in the $\mathrm{LHCb}$ experimental area. The aim of the detector is to perform high precision tracking of charged particles in the low polar angle of the LHCb experiment. The main areas of R\&D have been the optimization of the silicon sensor geometries, the development of a dedicated $\mathrm{LHCb}$ front-end readout chip and a fast digital optical readout link, and the design of light-weight solutions for the mechanical support and cooling of front-end electronics and silicon sensors.

The performance of the detector has been assessed using data collected during the LHC synchronization tests. An initial alignment of the detector with tracks has been performed. The efficiency of the detector has been proven to be good. The response of the detector to minimum ionizing particles in term of $\mathrm{S} / \mathrm{N}$ is compatible with the expectations from test-beams results. The knowledge and experience gained during these tests has helped enormously the understanding of the detector with colliding beams at the end of 2009 when the LHC restarted. The data from the collision is currently being used to improve the alignment of the sub-detectors, especially for the TT, using long tracks coming from the VELO.

\section{REFERENCES}

[1] D. Esperante et al., "The LHCb Silicon Tracker, first operational results”, CERN, Geneva, Conf. Rep., LHCb-CONF-2010-003, 2010.

[2] The LHCb Collaboration, "The LHCb detector at the LHC", Journal of Instrumentation, JINST 3 (2008), p. S08005.

[3] H. Voss, "The LHCb Silicon Tracker", Nucl. Instrum. Meth., vol. A549, pp. 44-48, 2005.

[4] J.v.Hunen, "The LHCb tracking system", Nucl. Instrum. Meth., vol. A572, pp. 149-153, 2007.

[5] S. Lochner, "Development, Optimisation and Characterisation of a Radiation Hard Mixed-Signal Readout Chip for LHCb", Ph.D. dissertation, Max-Planck Inst., Heidelberg Univ., Germany, 2006.

[6] A. Vollhardt, "An Optical Readout System for the LHCb Silicon Tracker", Ph.D. dissertation, Physik-Institut, Zurich Univ., Switzerland, 2005 .

[7] G. Haefeli et al., "The LHCb DAQ interface board TELL1", Nucl. Instrum. Meth., vol. A560, no. 2, pp. 494-502, 2006.

[8] O. Steinkamp, "Design and production of detector modules for the LHCb Silicon Tracker", Nucl. Instrum. Meth., vol. A579 pp. 736-741, 2007.

[9] D. Esperante, A. Vollhardt, "Design and development of the Control Board for the LHCb Silicon Tracker", CERN, Geneva, Tech. Rep., LHCb-2007-153, 2007.

[10] Z. Guzik, R. Jacobsson, B. Jost, "Driving the LHCb front-end readout", IEEE Trans. Nucl. Sci., vol. 51, no. 3, pp. 508-512, June 2004.

[11] R. Jacobsson, B. Jost, Z. Guzik, "Readout supervisor design specifications”, CERN, Geneva, Tech. Rep., LHCb-2001-012, 2001.

[12] G. Conti et al., "Inner Tracker Survey Strategy", CERN, Geneva, Tech. Rep., LHCb-2008-068, 2008.

[13] M. Needham, "First alignment of the Inner Tracker using data from the TI-8 sector test”, CERN, Geneva, Tech. Rep., LHCb-2009-030, 2009.

[14] L. Nicolas et al. "Tracking stations alignment with Kalman tracks at LHCb”, Proceedings of the IEEE Nucl. Sci. Symposium, Dresden, 2008.

[15] L. Nicolas and M. Needham, "Alignment of the Inner Tracker Stations Using First Data”, CERN, Geneva, Tech. Rep., LHCb-PUB-009-012, 2009 . 